\title{
RealScape-Metropolitan Fixed Assets Change Judgment by Pixel-by-Pixel Stereo Processing of Aerial Photographs
}

\author{
Hirokazu Koizumi, Hiroyuki Yagyu, Kazuaki Hashizume, \\ Toshiyuki Kamiya, Kazuo Kunieda, and Hideo Shimazu
}

\begin{abstract}
- The Tokyo Metropolitan Government, the largest municipality in Japan, routinely conducts building-change-identification work. Recently, Tokyo terminated its traditional visual-identification work, which had been used for 20 years, and shifted to a new automated system. This article introduces the Fixed Assets Change Judgment (FACJ) system and its core tool, RealScape. RealScape automatically detects changes in the height and color of buildings based on three-dimensional analysis of aerial photographs. It employs a unique pixelby-pixel stereo processing method and enables a foot-level precision for each building. RealScape detects building changes more accurately than visual judgment operations by humans and reduces the labor costs to one third of the traditional approach and the required judgment duration to about two weeks per $100 \mathrm{~km}^{2}$.
\end{abstract}

$\mathrm{T}$ This article describes the Fixed Assets Change Judgment (FACJ) system and its core tool, RealScape. RealScape automatically detects changes in the height and color of buildings based on three-dimensional analysis of aerial photographs. The three-dimensional analysis employs a pixel-by-pixel stereo processing method that calculates the height of each pixel in aerial photographs and thus enables precise difference detection between previous and current aerial photographs. The FACJ system reduces the labor costs to one third of the traditional approach and the required judgment duration to about two weeks per $100 \mathrm{~km}^{2}$. The FACJ system was experimentally used by the Tokyo Metropolitan Government for the first time in 2005. Since then, it has been used at its tax bureau every year to calculate the municipality's fixed-asset tax. After the success in Tokyo, other major city governments, including Osaka and Sapporo, have followed suit.

\section{Problem Description}

The Japanese fixed-property tax is imposed by municipalities on the owners of land, buildings, and depreciation assets (all hereinafter referred to as "fixed assets") on January 1 of every year by calculating the tax sum according to current asset values. For this purpose, the municipalities take aerial photographs every year on January 1 and compare the photographs with those of the previous year to identify building-change information (new construction, loss, enlargement, reform, reconstruction, work- 


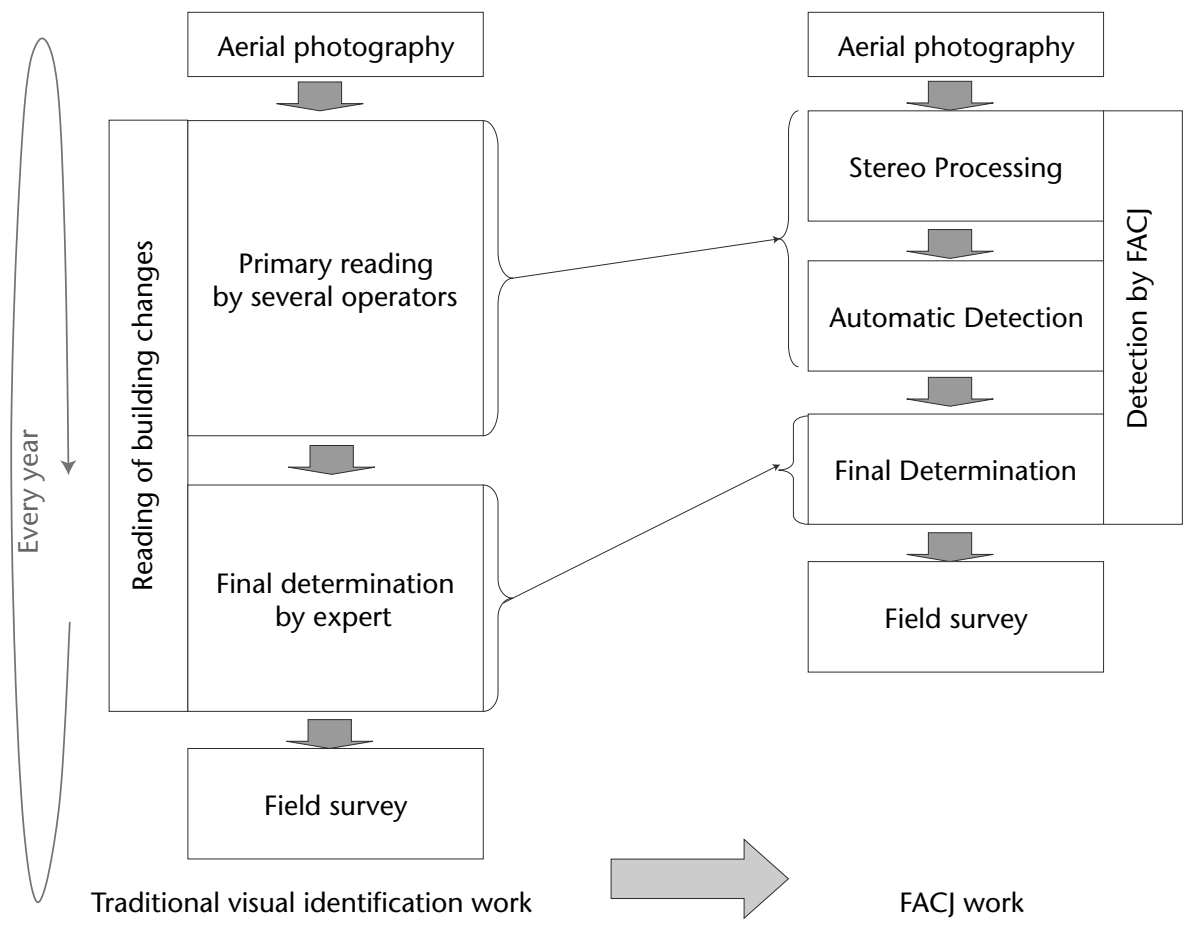

Figure 1. Building Change Identification Work Flow.

For imposing the fixed-property tax, municipalities in Japan survey building changes by aerial photographs every year. This identification work is contracted out to survey companies. FACJ represents a major conceptual shift from the traditional building-change-identification work flow.

in-progress, and so on). The identification of such changes is entrusted to survey companies who hire a large number of workers (figure 1, left). However, reliance on human labor has led to problems detailed in the following paragraphs.

\section{Huge Costs, and the Impossibility of Eliminating Human Judgment Errors}

It takes about 10 hours to read and interpret a single photograph, and the average municipality must perform this work for several hundred photographs. In addition, errors are not acceptable from the viewpoint of fair taxation, in particular, oversights in finding actual changes to buildings. Nevertheless, the current work done using the traditional system is dependent on the capabilities of individuals, so errors are unavoidable. In addition, in visual-identification work, attempts to prevent oversight errors are made by performing several read operations per area (figure 2), but this leads to a further increase in cost. Every photograph is tak- en over a scale that can cover an actual area of 800 by 600 meters or 500 by 600 meters (variable depending on the municipality), and every municipality has several hundred photographs that must be read. As a result, it is not rare for the personhours required for the photograph-reading operation to exceed 10,000 .

Under these circumstances, the incentives for the municipalities to overcome such challenges by automating or systematizing the photograph-reading work are now higher than ever. The criteria for the identification of changes are based on laws and guidelines issued by the Research Center for Property Assessment System. Specifically, the criteria are designed to detect height changes of 2 meters or more in an area of approximately 2 by 2 meters, and color changes in an area of approximately 2 by 2 meters. In other words, these criteria require the detection of any change in an area over 2 by 2 meters. Since these requirements set such a high hurdle for achieving automatic processing, the 


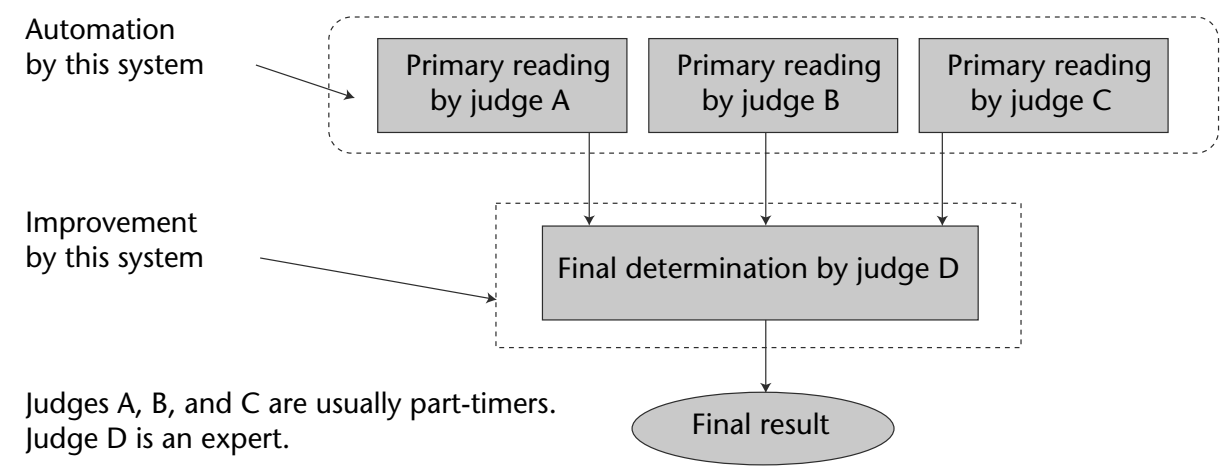

Figure 2. Work Flow.

In the traditional visual-identification work flow, several read operations are performed for each area to prevent oversight errors.

attempts made to automate this work have so far been limited to use of a few specific tools, and the available technology is still far from real systematization. The automation of this work involves the following problems.

\section{Height and Color Change Detection}

The height information may be obtained by aerial surveys using a laser profiler, but the laser profiler does not satisfy the area requirement because its resolution is too sparse to satisfy this requirement in normal use. Moreover, aerial survey devices are too expensive and their availability is limited for many municipalities.

Precise overlapping of previous and new photographs is required for detecting color changes. However, two aerial photographs are difficult to overlap and match satisfactorily because the angles of the buildings are usually different due to variances of the shooting conditions (shooting position, altitude, focal distance, and so on).

We have solved the height and color changedetection problems by adopting the method of pixel-by-pixel stereo processing, which makes it possible to obtain the height information of all pixels in aerial photographs and correct their positional information.

\section{Proposed Identification Work Flow}

FACJ provides a new concept of identification work flow for the annual detection of building changes (figure 1, right). The reading process for building change in the traditional identification work flow is replaced by the FACJ identification work flow. Here, stereo processing and an automatic detection process supplant the primary reading process now done by several human operators.
The final determination process by experts is almost the same, but a final determination tool is used in FACJ. Specifically, FACJ detects the building changes automatically and presents the user with a set of candidate changes, which are then validated by the user. The automation of the primary reading by stereo processing and the automatic detection reduce costs and time. In addition, this automation enhances the objectivity and fairness of tax assessment.

\section{Application Description}

The major component of the FACJ system is RealScape. Because RealScape is a general-purpose software package, the FACJ system is given some minor customization for the use of the tax bureau, such as data conversion and integration with other applications. In this article, we focus on RealScape, which consists of two modules (figure 3 ): the stereo processing module, and the changedetection module.

The stereo processing module inputs two aerial photographs into the computer, converts all of their pixels into three-dimensional information, and calculates the building height information within an accuracy of one meter. At the same time, it applies true orthorectification processing to correct for the inclinations of buildings in the photographs and to permit a precise overlapping of the previous and new photographs.

The change-detection module inputs the two (previous and new) orthorectification aerial photographs and the digital surface model (DSM) information and then detects changes in the shapes and colors of buildings and land. After that, the candidate changes are validated by the user to determine the final result of changes. 


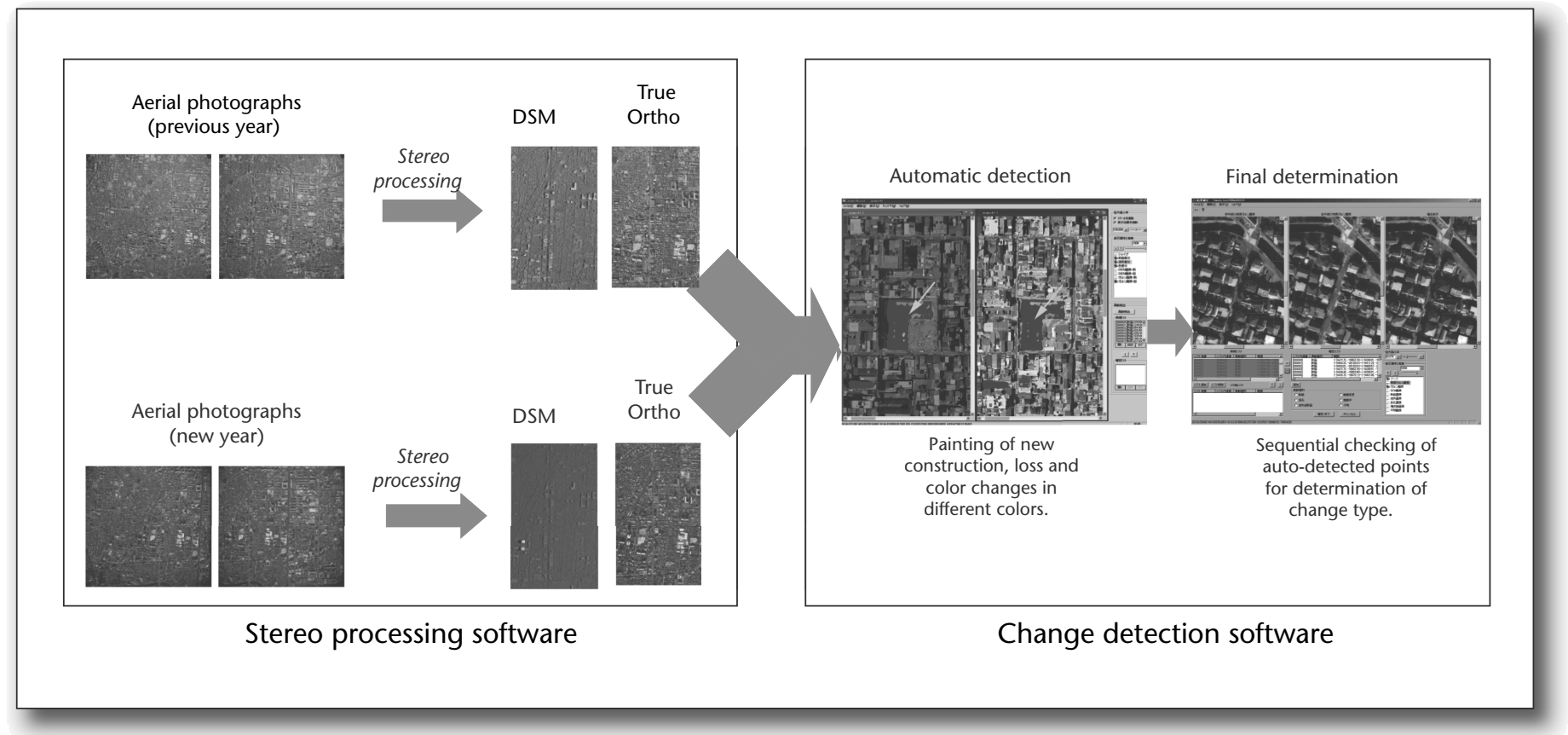

Figure 3. RealScape Modules.

RealScape consists of a stereo processing module that calculates building heights and performs orthorectification and a change-detection module that identifies changes in the images. DSM: digital surface model.

The target we set before designing this system was to improve through automation both the performance and accuracy of making judgments (prevention of human errors and detection of height changes that are difficult to detect visually). Even with the current technology level, it is difficult to achieve a perfectly error-free reading and judgment automatically. Therefore, we decided to apply judgment using automated software in the primary reading and to carry out the final reading only with the tools for accurate judgment. According to actual data, the number of buildings shot per photograph is between 3000 and 4000, and the annual percentage of buildings subjected to changes is 3 percent to 5 percent. Accordingly, the average number of changed buildings in a photograph is around 150 . Since the aim of primary reading in the traditional visual judgment work is to select 500 to 600 candidate buildings among the 3,000 buildings in a photograph, we set the target performance of our automatic judgment system, which is to be used in the primary reading, at a level of judgment capability similar to that of the traditional primary reading stage.

\section{Stereo Processing of Aerial Photographs}

AI technology is used in the stereo processing module. Specifically, various image-processing techniques such as customized dynamic program- ming (DP) matching are adopted. Figure 4 shows an outline of the stereo processing module.

\section{Input Data}

The input images can be analog or digital aerial photographs similar to those used in the traditional change-judgment system. Each of these photographs consists of a series of picture frames taken of an area, with about a 60 percent overlap between the frames. This system executes stereo processing by assigning two adjacent picture frames as the left and right images and obtains the height information of all pixels in the overlapped area.

\section{Relative Orientation}

Unlike photographs in which images are taken using a stereo camera that can be calibrated every time before use, aerial photographs are taken using various camera attitudes. This procedure makes it necessary to perform image rectification, which is a method for parallelizing a pair of images. For this purpose, the system rectifies input images by using the external orientation parameter. Then, orientations of the input images are aligned.

\section{Pixel-by-Pixel Stereo Matching}

The main task in stereo matching is to identify the corresponding points in the left and right images. When the left and right images are made parallel by the relative orientation, all of the subjects are located on the same scanning line number in the 


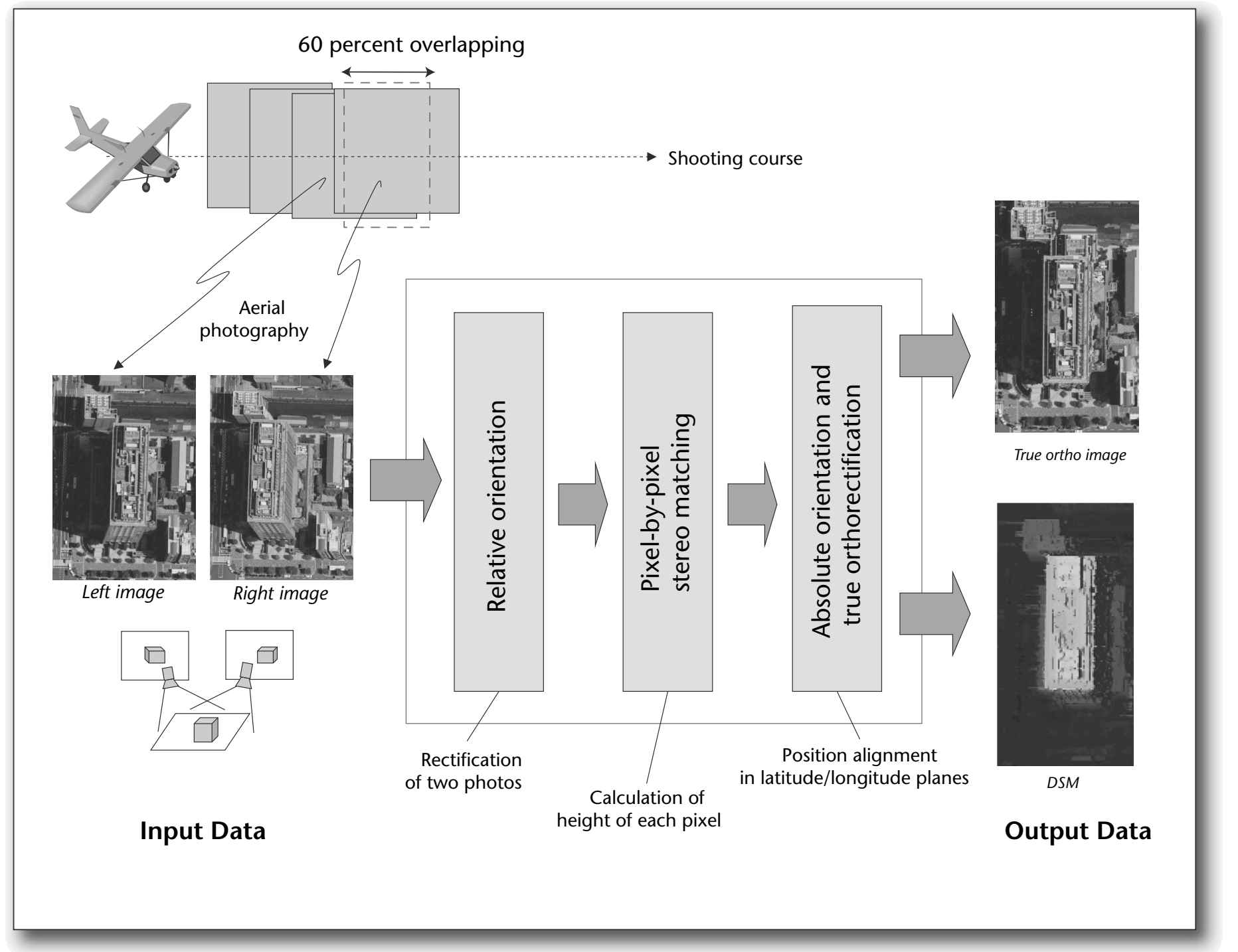

Figure 4. Stereo Processing Module.

The stereo processing module consists of a relative orientation step that rectifies stereo images for parallelizing, a pixel-by-pixel stereo matching step that identifies the corresponding points of stereo images, and an absolute orientation and true orthorectification step that converts the image.

left and right images. Therefore, the search for corresponding points can be limited to a single dimension. We adopted the DP matching method for the search, which uses the cross-correlation as the evaluation value and outputs the DSM after processing. The single dimension search and the DP matching reduce calculation time, which is important because the input images are extremely large (for example 7500 by 11,500 pixels). To acquire a precise DSM of sharp height change, such as the buildings in a downtown district, we customized the DP algorithm to permit discontinuous correspondence. In order to obtain high-quality DSM, it is necessary to select the cross-correlation parameters, such as the window sizes and threshold values, optimally according to the scale and type of input images. Because the selection of these parameters requires a certain degree of experience, we provided the system with parameter settings that are optimized according to the type of processed images, thus allowing the user to perform optimum processing simply by selecting one of the parameter settings.

\section{Absolute Orientation}

Since processing for pixel-by-pixel stereo matching is performed in the image coordinate space, it eventually becomes necessary to compare the matched correspondence of the photograph and the DSM with the latitude and longitude of the land location in this procedure. At the same time, a conversion of the parallax values obtained by the stereo matching of the altitude value is also performed. 


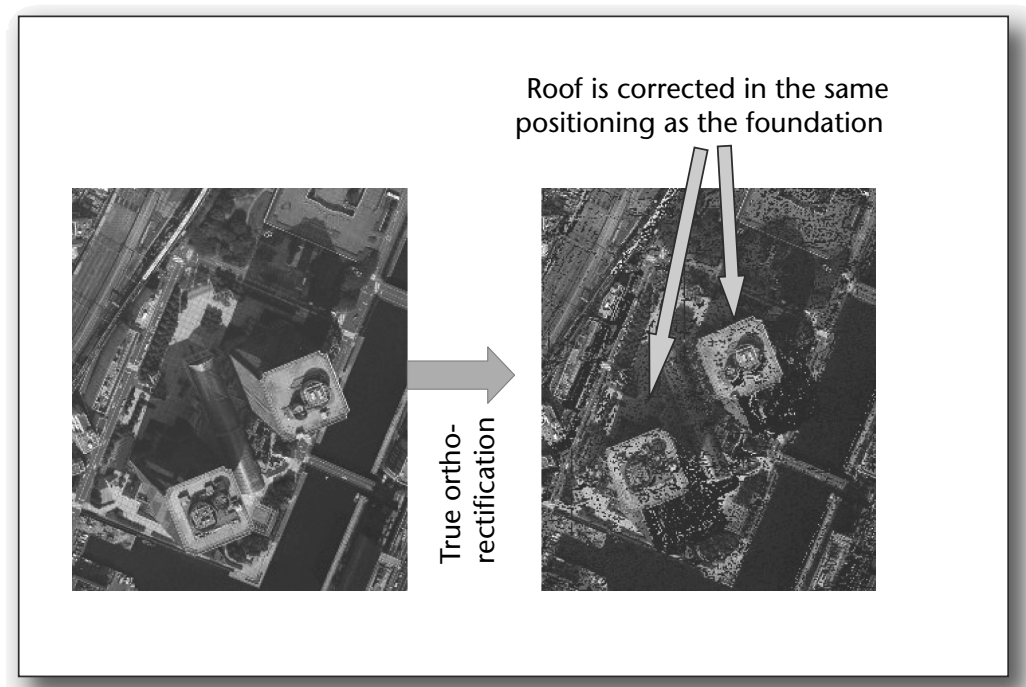

Figure 5. True Orthorectification.

True orthorectification corrects the roof surfaces of all buildings to their real positions.

\section{True Orthorectification}

Stereo processing requires a large number of calculations. Therefore, traditional aerial survey software generally obtains the altitude information only for the characteristic points and the contour lines of topography and buildings, and it then applies interpolation for other points. This has resulted in problems such as dealing with buildings that lack contours and are undistinguishable from the ground, consequently remaining inclined in the images. On the other hand, RealScape can determine the absolute positions of all pixels because its stereo processing offers the height information of all pixels without the need for contour information. We call this processing method the "True Orthorectification" method. True ortho images show the roof surfaces of all buildings in their real positions without tilting (figure 5). This makes it possible to overlap a photograph precisely onto a map or to overlap two photographs taken under different shooting conditions. Figure 6

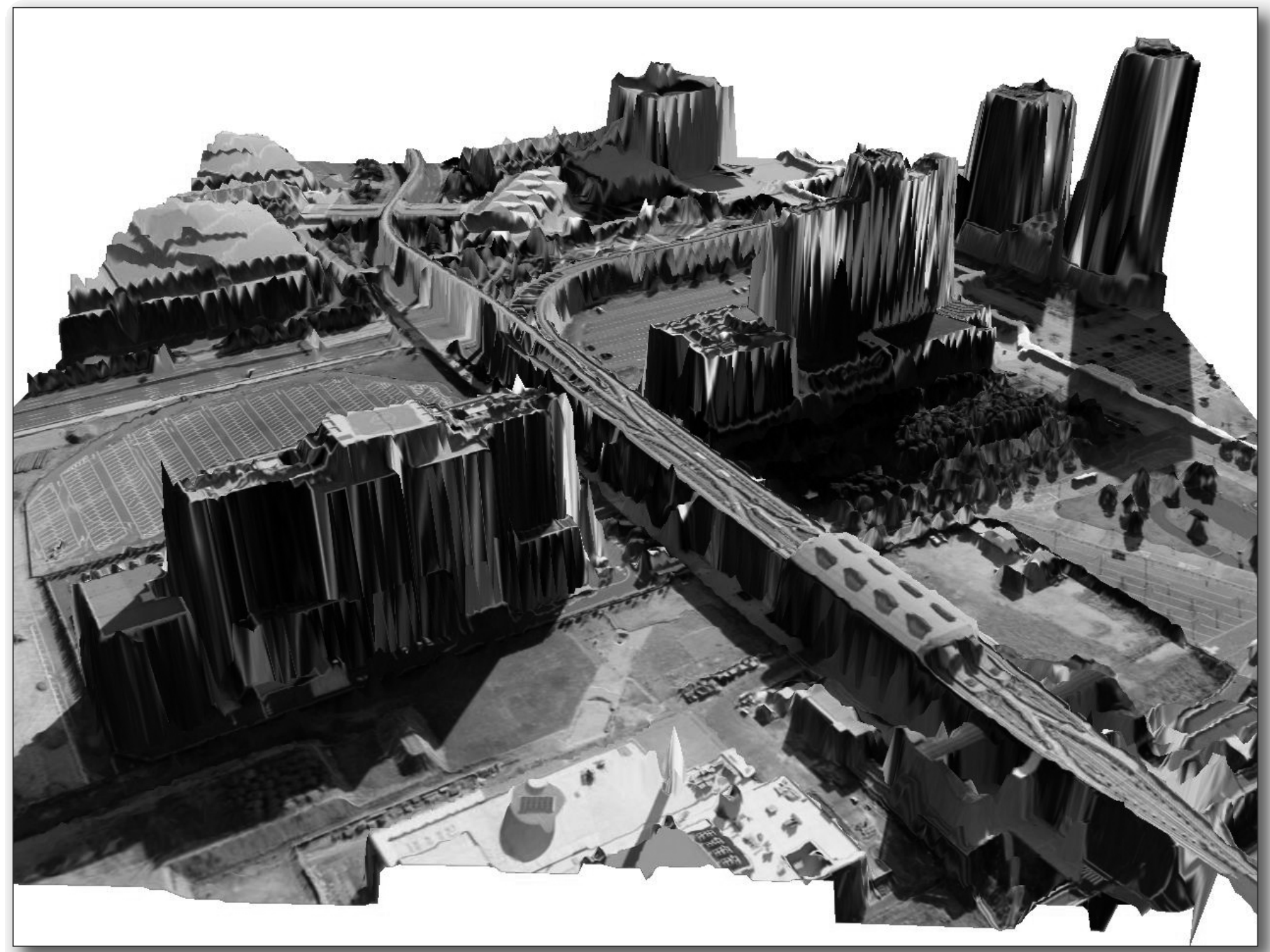

Figure 6. RealScape can Generate a Three-Dimensional View. 


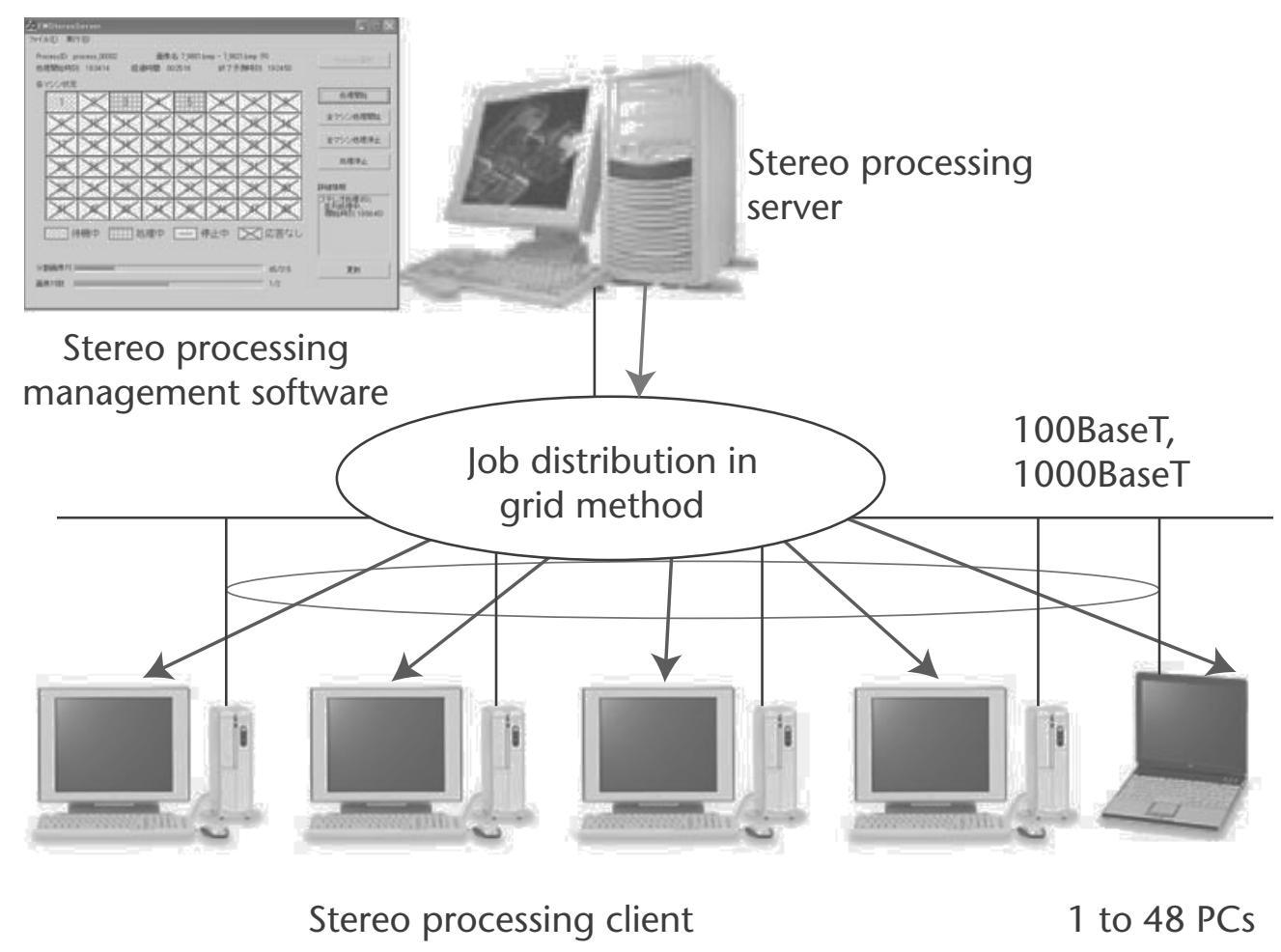

Figure 7. RealScape Hardware Configuration.

The RealScape hardware configuration features a server-and-client system for concurrent execution of stereo processing.

shows an example three-dimensional view of downtown Tokyo generated by RealScape without any manual editing.

\section{Hardware Configuration}

RealScape runs on multiple PCs (figure 7). It includes a server-and-client system for concurrent execution of stereo processing using a grid computing architecture. It also contains software for use in a series of operations. The system can perform the work of generating a DSM from aerial photographs. The clients are composed of 1 to 48 PCs, which can each join or leave the stereo processing dynamically, allowing a flexible system configuration.

\section{Change Detection of Buildings}

The change-detection software inputs both previous and new color images and the DSMs obtained as a result of stereo processing, and it then detects any changes in them. This software is composed of an automatic detection tool for the primary reading operation and the final determination tool for supporting the final judgments by experts (figure 8).

\section{Automatic Detection Tool}

Absolute orientation and orthorectification of stereo processing create height and color data that have precise geolocation information for each pixel. Therefore, simple pixel-by-pixel comparison between the previous and current data is sufficient to detect land-use changes. The automatic detection tool was designed to specifically analyze and detect changes in buildings such as new construction, loss, or color changes. For the altitude changes, this software refers to both previous and new DSMs and then detects an increase in height as a new construction or a decrease in height as a loss. For the color changes, it refers to the true ortho color images and detects changes above threshold values as color changes. Since it is difficult for users to specify the threshold value for color change, the system at first presents the threshold calculated automatically by considering the illumination difference between two images. Here, all of the areas that were detected with color changes cannot be interpreted as actual changes because objects of a certain size, such as automobiles, could have been falsely detected as areas with color changes. Therefore, our system also refers to 

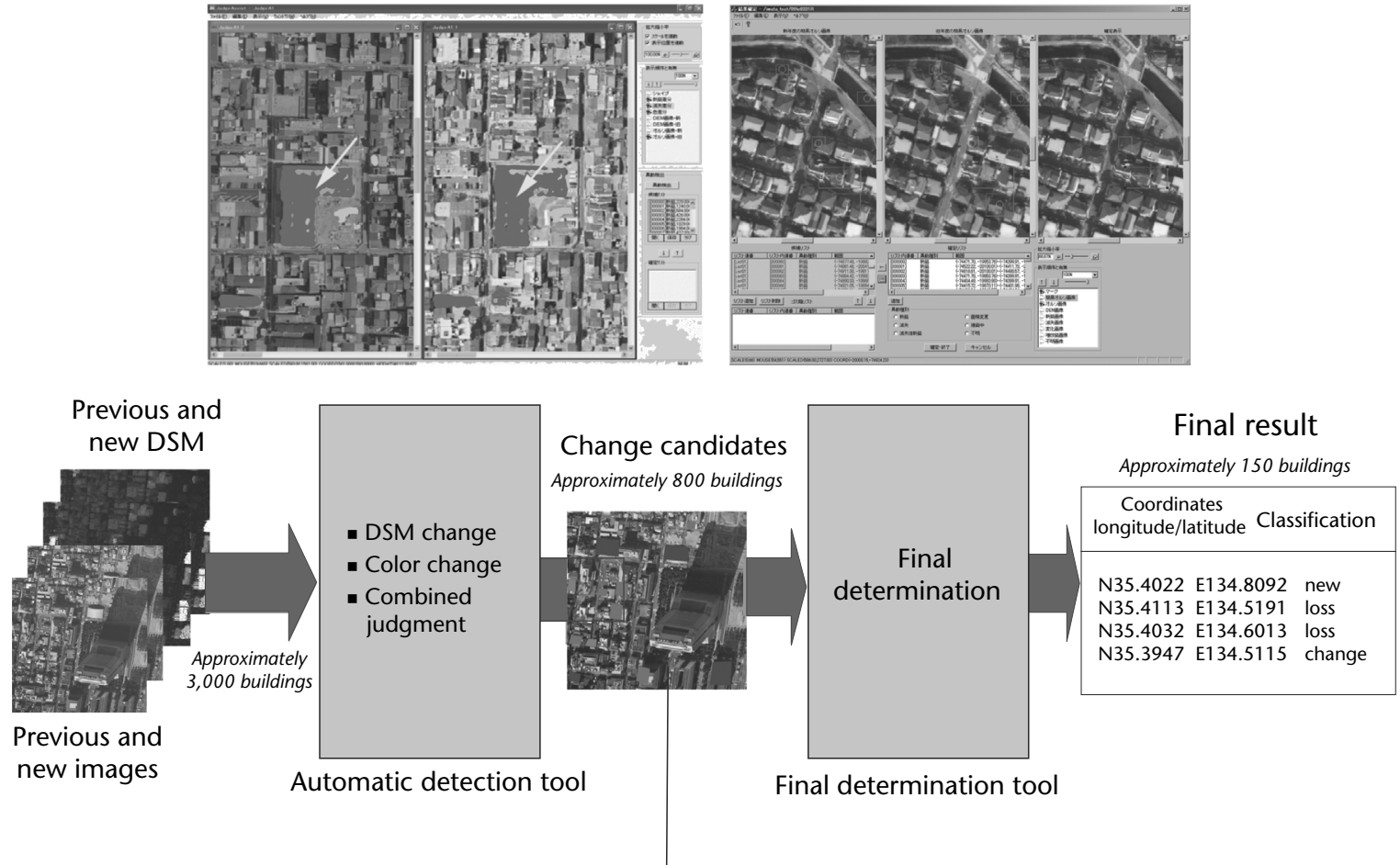

Final determination tool

Detection by categorizing into new construction, loss and color change

Figure 8. Change-Detection Module.

The change-detection module is composed of an automatic detection tool that automatically detects building changes and a final determination tool that assists experts performing the final judgments.

the DSM to eliminate color changes in areas with a height of below two meters from the ground. The results obtained with this tool are output as a map image showing new construction, losses, and color changes by particular painted pixels, and the center coordinates (latitude and longitude) of each change area that are detected as a mass by labeling.

\section{Final Determination Tool}

The final determination tool is designed to assist experts performing the final judgments. The colored map and center coordinate list obtained from the automatic detection tool are taken as input for this tool. While the results of automatic detection indicate three types of changes (new construction, loss, and color change), the final determination tool is also capable of more detailed categorization of change types (new construction after loss, works in progress, and so on) according to the requirements of municipalities.

\section{Performance Evaluations}

We evaluated the accuracy of the DSM for analog and digital photographs, as well as the accuracy of change detection.

We evaluated the horizontal and vertical accuracies of a DSM created from analog aerial photographs at 40 points in the mountains, hills, and urban areas by comparing the DSM with field surveys. Table 1 shows the specifications of aerial photographs and field surveys. Table 2 shows the results, 119 centimeter horizontal accuracy and 49 centimeter vertical accuracy. This satisfies the requirements defined in the public survey regulations.

We evaluated the vertical accuracy of the DSM created from digital aerial photographs by comparing it with field surveys and laser profiler data. Table 3 shows specifications and table 4 shows the results, which were 15.9 centimeter vertical accuracy for the DSM and 12.4 centimeter vertical accu- 


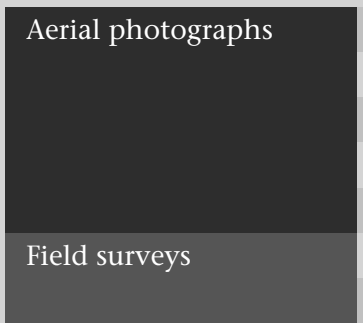

Scale

Spatial resolution

Overlap rate

Focal length

Scan resolution

Survey method

Number of points
$1 / 10,000$

$21 \mathrm{~cm}$

Overlap: 60\%; Sidelap: 30\%

$152.96 \mathrm{~mm}$

$1,200 \mathrm{dpi}$

Network-based RTK-GPS

40 points

Table 1. Specifications of Aerial Photographs and Field Surveys.

\begin{tabular}{lcc} 
& Horizontal accuracy & Vertical accuracy \\
\hline DSM & $119 \mathrm{~cm}$ & $49 \mathrm{~cm}$ \\
Public survey regulation & Within $175 \mathrm{~cm}$ & Within $67 \mathrm{~cm}$
\end{tabular}

Table 2. Accuracy of DSM Created from Analog Aerial Photographs.

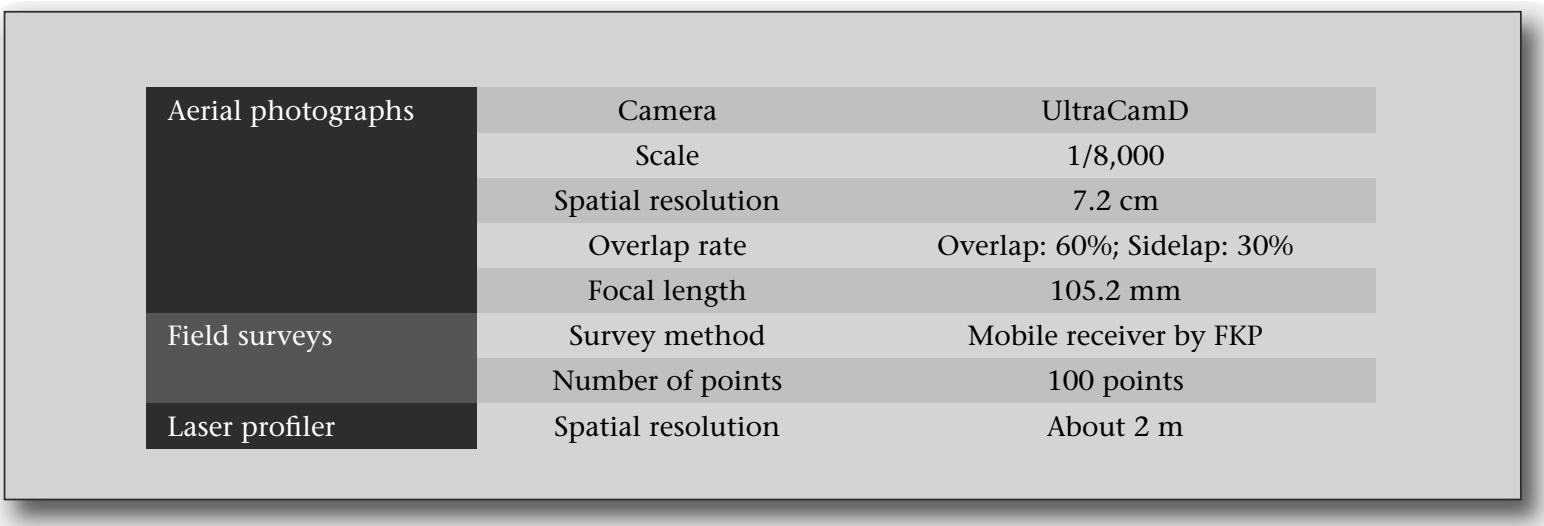

Table 3. Specifications of Aerial Photograph, Field Survey, and Laser Profiler Data.

$\begin{array}{lcc} & \text { Vertical accuracy } & \text { Resolution } \\ \text { DSM } & 15.9 \mathrm{~cm} & 7.2 \mathrm{~cm} \\ \text { Laser profiler data } & 12.4 \mathrm{~cm} & \text { About } 2 \mathrm{~m}\end{array}$

Table 4. Accuracy of DSM Created from Digital Aerial Photographs

racy for laser profiler data. The DSM was nearly equal to laser profiler data in vertical accuracy. On the other hand, the resolution of laser profiler data was only about 2 meters, while that of the DSM was 7.2 centimeters, which is equal to the resolution of an aerial photograph. Therefore, the DSM created by RealScape had much higher resolution than laser profiler data.
In a 660 by 1200 meter flat-land area, which included 1,636 buildings in a certain city, we evaluated the change-detection accuracy of RealScape by comparing the results of RealScape with those of human visual identification. Table 5 shows specifications of the photographs. For evaluation, we checked whether the detected changes were true changes or not by careful visual identification. 


$\begin{array}{lcc}\text { Year } & 2006 & 2004 \\ \text { Camera } & \text { UCD (digital) } & \text { RC30 (analog) } \\ \text { Scale } & 1 / 8,000 & 1 / 8,000 \\ \text { Scanning } & - & 21 \mu \mathrm{m} \\ \text { Spatial resolution } & 7.2 \mathrm{~cm} & 16.8 \mathrm{~cm} \\ \text { Overlap rate } & 80 \% & 60 \%\end{array}$

Table 5. Specifications of Photographs.

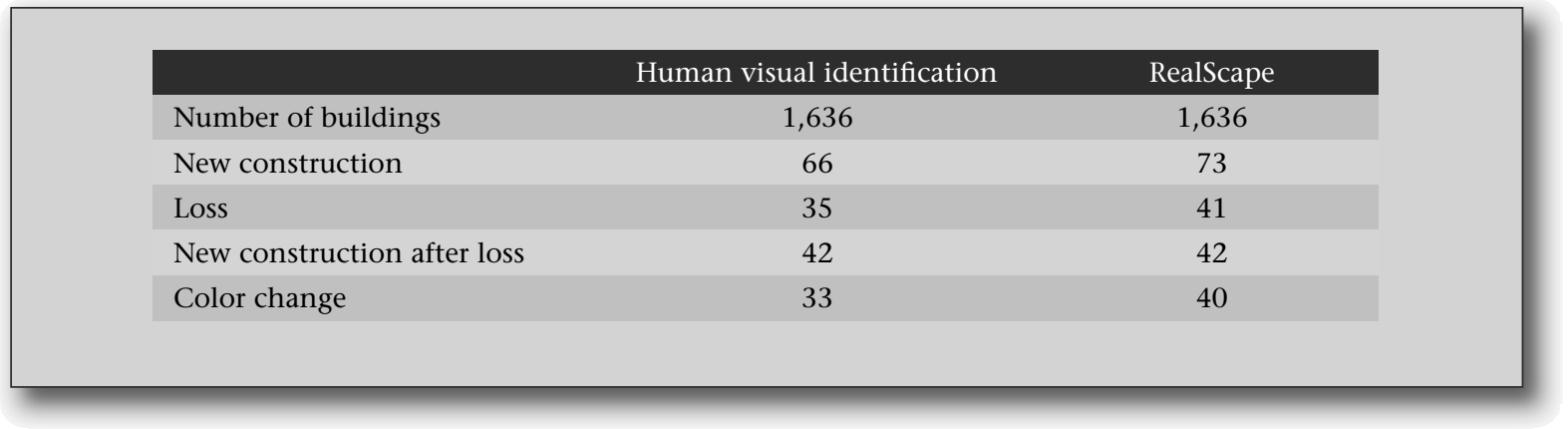

Table 6. Evaluation Results of Change Detection.

From the results (table 6), RealScape detected all of the changes in new construction, loss, and new construction after loss that were detected by visual identification. Furthermore, RealScape additionally detected seven new construction changes and six loss changes not detected by the human visual identification. Figure 9 shows an example of change that RealScape detected as loss and that the human visual identification failed to detect. In the human visual identification, the oversight of a building occurred if the color of a roof in one photograph was very similar to that of the ground color in another photograph. Even in such a case, RealScape correctly judged the change as loss by their height difference.

Figure 10 shows an example of change that RealScape detected as a newly constructed building but that the human visual identification failed to detect. In the visual identification, it seemed that the area was judged as a parking space because the color of the roof in a photograph was similar to that of asphalt. RealScape judged it correctly as new construction. As for color change detection, RealScape detected ten color-changed buildings not detected by the human visual identification and detected 30 color-changed buildings out of 33 detected by the human visual identification. The three additional changes detected by the human visual identification were mistakes, since the humans concluded that the heights were different when they were actually the same. This evaluation reveals that change detection by RealScape has almost equal accuracy to that of visual identification. Furthermore, RealScape is able to detect height change, which is difficult to judge by human visual identification.

\section{Application Development, Use, and Payoff}

It took two years for four of us to develop RealScape from 2003. We developed a prototype in the first year and the software package in the second year. In fiscal 2005, we experimentally deployed it in the fixed-assets change-detection work with the support of the Tokyo Metropolitan Government. We created the full-scale DSM of 2800 photographs (on-ground resolution: 12.5 centimeters) of the Tokyo metropolitan area and obtained confirmation from the Japan Association of Surveyors that the results were within the standard error tolerance of 50 centimeter horizontal accuracy and 100 centimeter vertical accuracy.

For detecting change, we conducted comparisons of 10 photographed districts in Shinagawa Ward, which is in the central part of downtown Tokyo, and verified this approach by comparing the results with those of independently conducted visual judgment operations. Consequently, we found that height changes were detected for horizontal or vertical change by 2 meters or more, in areas of 1 by 2 meters or wider, and color changes were detected in areas of 2 by 2 meters or wider. 

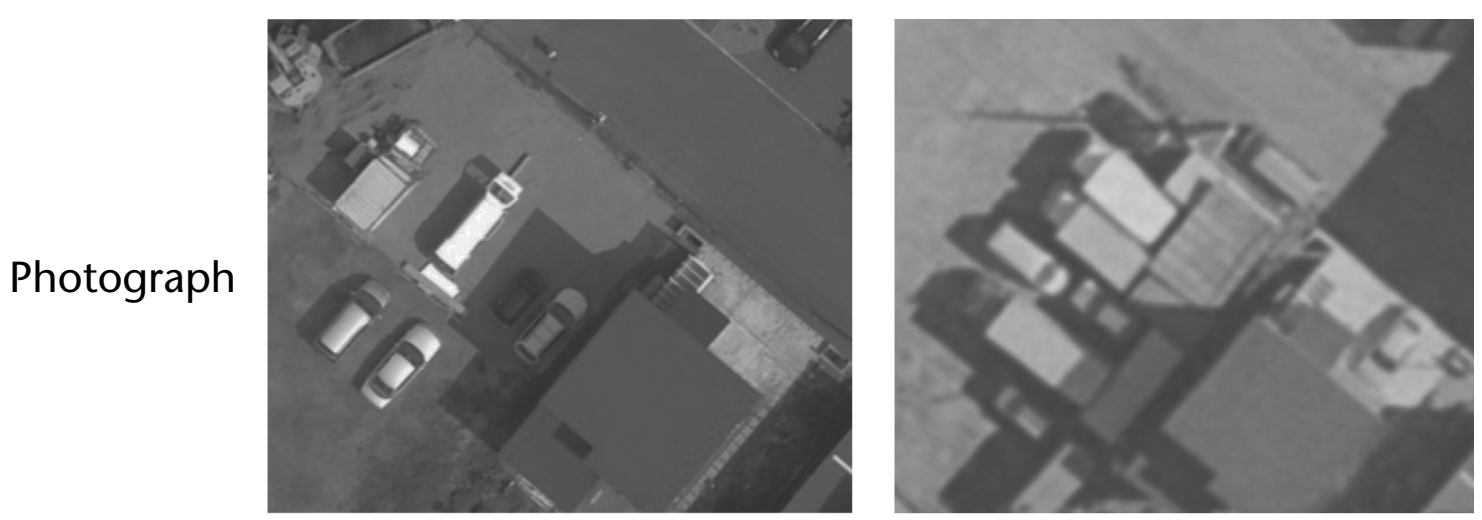

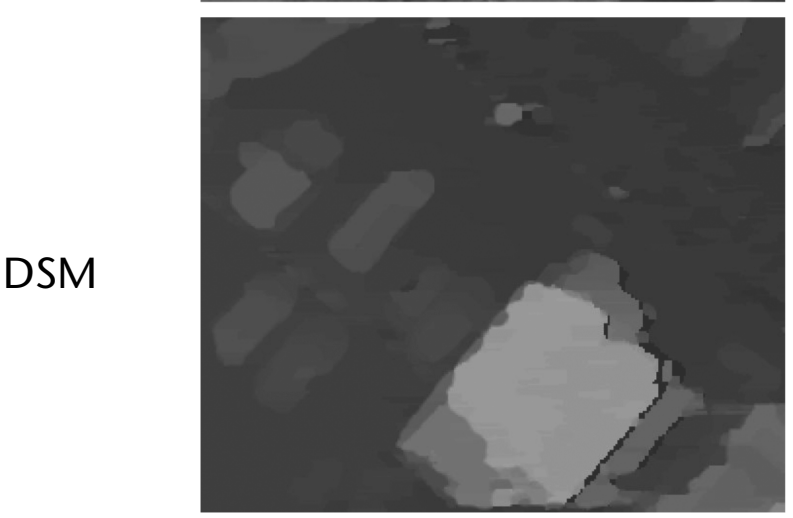

New Year

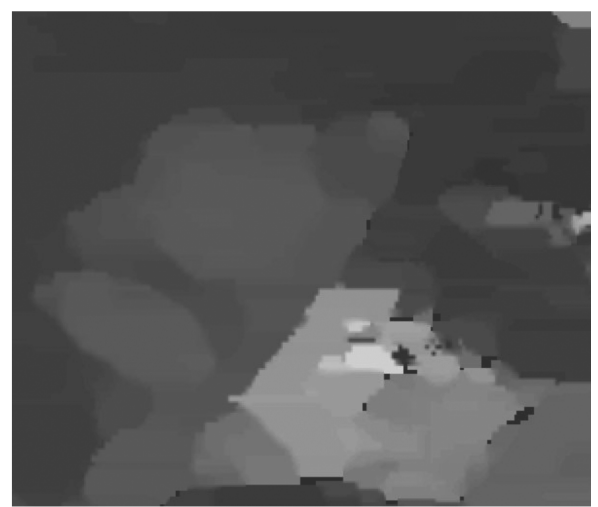

Previous Year

Figure 9. RealScape Detects Image Loss.

RealScape detected a loss in the image, but visual identification missed it.

Since it is estimated that the time taken for the manual final determination operation is 2 to 3 hours for each pair of images, our system can reduce operation time by about 60 percent from the time needed for the traditional judgment process by one person as shown in figure 11. This time-saving effect may be even larger if our system is compared to the traditional approach of using several read operations in each area.

This section describes the first RealScape deployment in Tokyo in 2006. The total area of Tokyo's 23 wards is about 621 square kilometers. To achieve the right coverage for our fixed-asset change detection, the total area was divided into four blocks. We used a total of 2800 aerial photographs for this area, or about 700 aerial photos per block. Four survey companies were contracted to work on one block each. The survey companies applied
RealScape software to perform their change detection using the following work flow: (1) preparation, 2) trial run, and (3) change detection.

In preparation, the survey companies were introduced to RealScape and its usage through a two-day training course we ran. Tokyo Metropolitan Government requested a trial run to confirm the survey companies' capabilities before the actual change-detection effort. The government wanted to ensure that these companies could successfully create the data according to the required specifications using RealScape. Upon a successful trial run, the survey companies conducted change detection on January 1 . They processed the aerial photography and triangulation data for about one week. Then the companies performed stereo processing for two to three weeks. Following this, they executed change detection (including automatic 

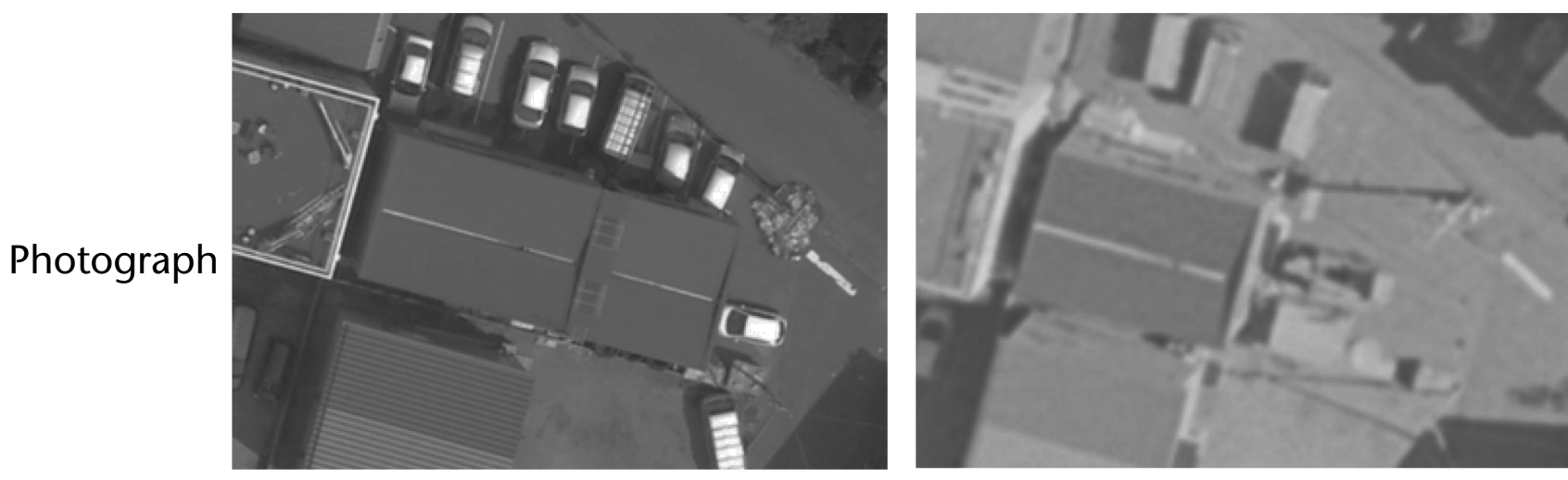

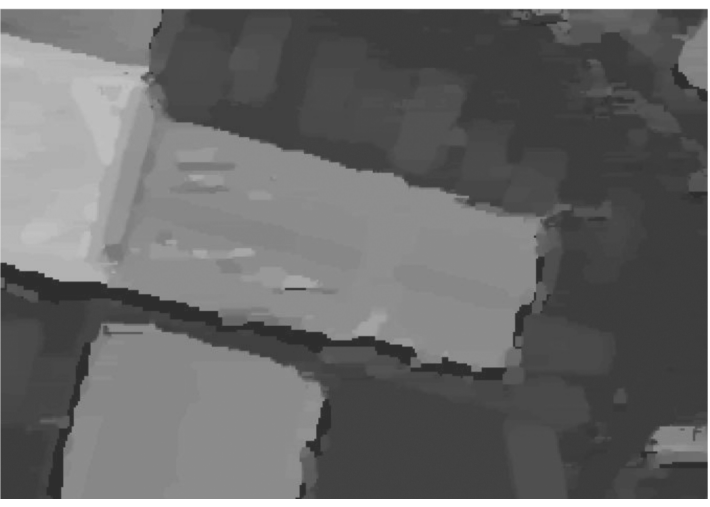

New Year

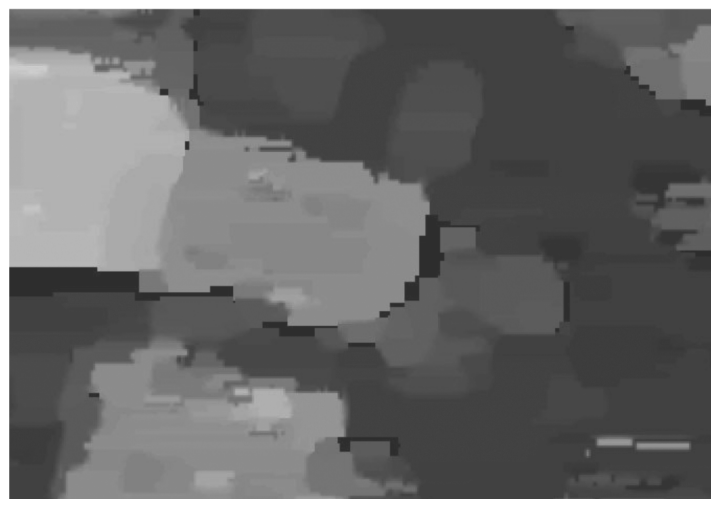

Previous Year

Figure 10. RealScape Detects New Construction.

RealScape detected new construction in the image, but visual identification judged it as loss.

detection and final reporting) for one month. In total, the survey companies required an average of two months to complete all building change reports, while this took three months by the traditional visual identification work flow.

Normally (and without Tokyo's stricter requirements), survey companies can simplify the work flow to reduce operating costs. However, as with the Tokyo case, highly accurate detection results enable municipalities to properly assess property taxes with greater accuracy and efficiency.

Tokyo has repeated this change-detection project using elevation data annually since 2007 . The city of Tokyo has gained many benefits, including reduced costs, better and more accurate change detection, and a faster tax assessment process since the introduction of RealScape.

Municipalities in Japan do not announce the actual amount of changes in tax revenue. But informally, we have heard that building changes not detected by the traditional way were newly detected by the proposed method. Therefore, FACJ can help to maximize collected tax income. Moreover, the use of FACJ can significantly reduce labor costs. This project model will be beneficial for any government tax assessment entity.

\section{Maintenance}

The FACJ systems are running internally at different survey companies that use the RealScape software package. Since RealScape is a self-contained software package, many traditional maintenance tasks, such as data backup, are provided in self-service mode. For the stereo processing part, no real maintenance is needed since the rules and algorithms are static. However, the time needed for the stereo processing is still large. For example, it takes 30 minutes for the pixel-by-pixel stereo matching when using 10 client PCs (Core2 Duo, $3.33 \mathrm{GHz}$ ). Therefore, we are striving to improve the algorithm to shorten this time. 


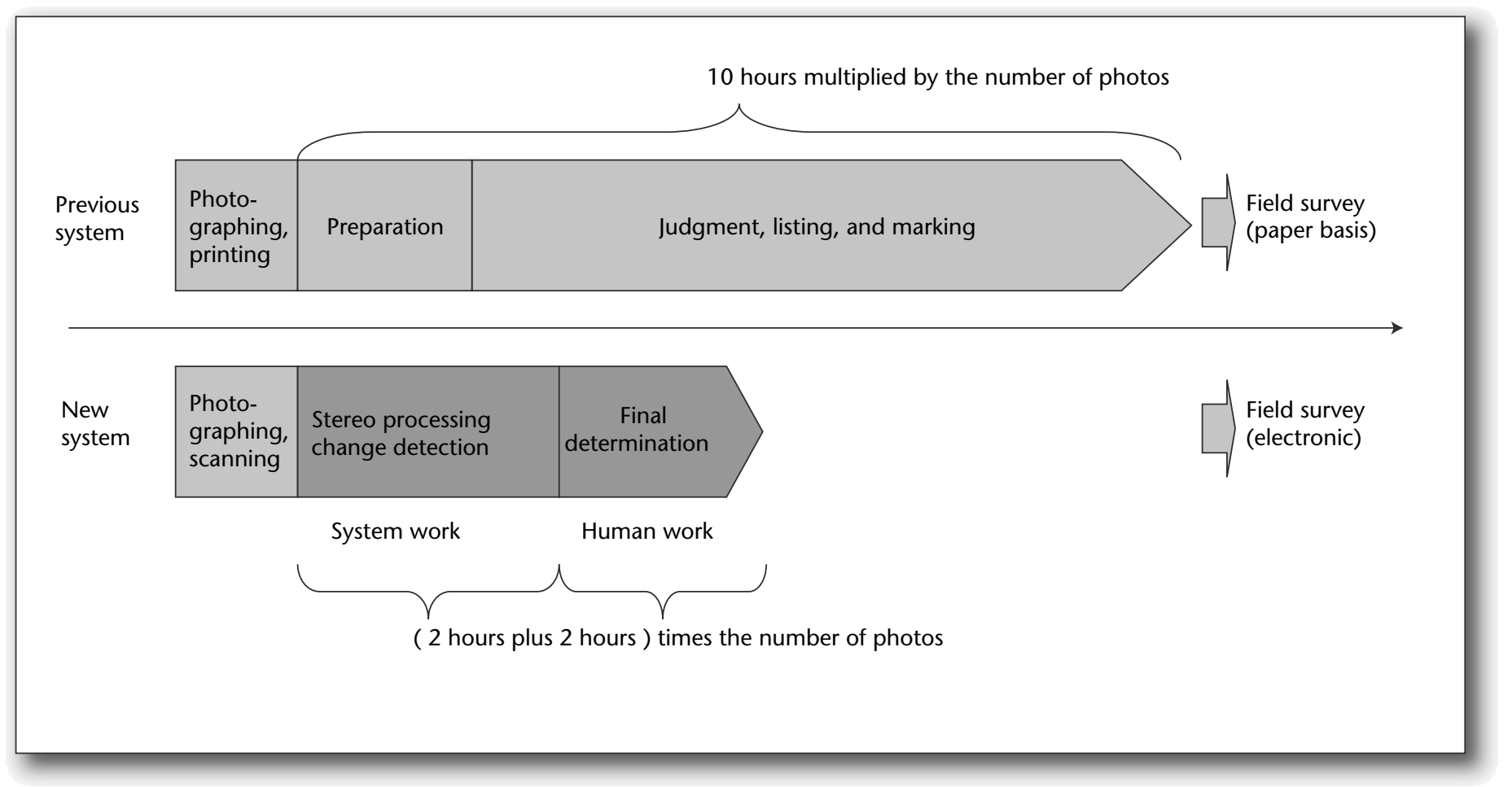

Figure 11. Operation Time Reduction.

RealScape can reduce operation time by about 60 percent compared to the time needed for the traditional judgment process.

\section{Comparison with Previous Works}

Change detection of buildings is an important task that has been addressed in many studies. Lillestrand (1972) compared pixel intensity of images taken at different times. Comparing intensity values is not very effective because such changes don't necessarily reflect actual changes in shape but could be caused by changes in viewing position, illumination conditions, and seasonal variations. Carlotto (1977) first predicted pixel intensities by extrapolating the changes that were seen in a sequence of satellite images taken from the same position. Pixels whose intensities did not match the predicted values were judged as changes. However, this method did not distinguish among building changes, parallax, and shadow. Huertas and Nevatia (1998) created three-dimensional wireframes of buildings from previous stereo image pairs and then projected the three-dimensional wireframes onto the current image. Building changes were detected by analyzing the overlap of the three-dimensional wireframes and the edges of current image objects.

The ATOMI project (Eidenbenz, Kaeser, and Baltsavias 2000; Niederost, 2000) was aimed at detecting changes and enhancing planimetric accuracy from the 5-meter level to the 1-meter level based on colored aerial photos, a digital elevation model (DEM), and a DSM. The ATOMI update work flow used the DSM as the primary data source, and it used the image information primarily to discern manmade objects from natural objects. Vogtle's approach used airborne laser scanning data (Vogtle and Steinem 2004). Changes were detected by comparison of DSMs, which were acquired at two different times. To eliminate nonbuilding objects, a segmentation procedure was used before the comparison.

Although it was necessary to get 50 centimeter horizontal and 1 meter vertical accuracy for our specific application, the aforementioned approaches had not achieved the required accuracies.

\section{Conclusions}

This article described the Fixed Assets Change Judgment system and its core tool, RealScape. Recently, Tokyo terminated its traditional visual identification work that had been in use for 20 years and shifted to the FACJ system. Because this deployment of RealScape had an impact on both the municipality community and the survey industries, after Tokyo's success, Osaka (Japan's third-largest city with a population of 2.6 million), Sapporo (Japan's fifth-largest city with a population of 1.8 million), and several other city governments followed suit. It is possible that the municipalities contract out the three-dimensional 
data-creation work and change-detection work separately, but FACJ is used for both jobs in Tokyo, Osaka, and Sapporo.

The challenge of this application was to achieve precise calculation of height and geographic location of each pixel by stereo processing. However, this deployment was also required to process several thousand large images (for example, 7,500 by 11,500 pixels) within the defined period at low cost. Therefore, we adopted pixel-by-pixel DP matching, which permits discontinuous correspondence, as the stereo matching method for calculating the height and geographic location of each pixel. By using this method, it is possible to reduce the amount of calculation through precise reproduction of sharp height change in features such as the buildings in a downtown district.

In the traditional work flow, it is necessary to identify the building changes by visually comparing photographs taken in two years and making correspondence to places and buildings. But this method's pixel-by-pixel processing makes it possible to match the positions of lands and buildings of different years in the manner of overlapping with a map and, moreover, to identify the changes in color and height by image processing (differential process), which is not dependent on human perception. Furthermore, we adopted a grid computing architecture to reduce processing time. By exploiting the parallelization of the stereo processing with the system's architecture, the processing time can be greatly reduced.

\section{Acknowledgements}

We would like to thank Shinji Sawada, Minoru Kawano, Sachio Michiya, Fumito Yamasaki, and Nobuyuki Nishimura for their contributions to this successful deployment. We also thank Ted Nguyen and Amanda Finch for their invaluable comments to improve this document.

\section{References}

Carlotto, M. 1997. Detection and Analysis of Change in Remotely Sensed Imagery with Application to Wide Area Surveillance. IEEE Transaction on Image Processing 6(1) 189-202.

Eidenbenz, C.; Kaeser, C.; and Baltsavias, E. 2000. ATOMI-Automated Reconstruction of Topographic Objects from Aerial Images Using Vectorized Map Information. International Archives of Photogrammetric and Remote Sensing XXIII, 8. Amsterdam, The Netherlands: International Society for Photogrammetry and Remote Sensing.

Huertas, A., and Nevatia, R. 1998. Detecting Changes in Aerial Views of Man-Made Structures. In Proceedings of the Sixth International Conference on Computer Vision, 73-80. Los Alamitos, CA: IEEE Computer Society.

Lillestrand, R. 1972. Techniques for Change Detection. IEEE Transactions on Computers 21(7): 654-659.

Niederost, M. 2000. Reliable Reconstruction of Buildings for Digital Map Revision. International Archives of Pho- togrammetric and Remote Sensing XXIIII, 8. Amsterdam, The Netherlands: International Society for Photogrammetry and Remote Sensing.

Vogtle, T., and Steinem, E. 2004. Detection and Recognition of Changes in Building Geometry Derived from Multitemporal Laser Scanning Data. International Archives of the Photogrammetry, Remote Sensing and Spatial Information Sciences, Vol. 34, Part XXX. Amsterdam, The Netherlands: International Society for Photogrammetry and Remote Sensing.

Hirokazu Koizumi is an assistant manager of System Technology Laboratory, NEC System Technologies, Ltd. He received an M.E. degree in information and communication engineering from the University of Tokyo in 2000. His research interests are image processing, computer vision, and pattern recognition.

Hiroyuki Yagyu is an assistant manager of the System Technology Laboratory, NEC System Technologies, Ltd. He received a B.E. degree in electronics engineering from the Osaka Electro-Communication University in 1985.

Kazuaki Hashizume is an assistant manager of System Technology Laboratory, NEC System Technologies, Ltd. He received a bachelor of economics degree from Osaka Sangyo University.

Toshiyuki Kamiya is a manager of System Technology Laboratory, NEC System Technologies, Ltd., and is responsible for the RealScape product line. He received an M.E. degree in electronics engineering from Kyoto University in 1992. He joined the Research Laboratory in NEC Corporation and spent 17 years researching visual interfaces, image processing, and knowledge management. His current research interests are image processing, computer vision, and pattern recognition.

Kazuo Kunieda is a senior manager of C\&C Innovation Research Laboratories, NEC Corporation. He received the B.E., M.E., and D.E. degrees in information science from Kyoto University in 1987, 1989, and 1995, respectively. From 2003 to 2006, he joined NEC System Technologies, Ltd., and worked on the RealScape project. His current research focus includes computational models of human behavior for improving creativity. He is a member of the Information Processing Society of Japan and the Institute of Electronics, Information, and Communication Engineers of Japan.

Hideo Shimazu is a research director of System Technology Laboratory, NEC System Technologies, Ltd. (Japan). He received his B.S., M.S., and Ph.D. degrees from Keio University. His work has focused on artificial intelligence, ubiquitous computing, information security, and robots (his world's first Sommelier robot has been recorded in the Guinness book of world records). He received the JSAI Distinguished Service Award from the Japanese Society of Artificial Intelligence in 2004. 